\title{
Efficient Skin Region Segmentation using Low Complexity Fuzzy Decision Tree Model
}

\author{
Rajen B. Bhatt ${ }^{\# 1}$, Abhinav Dhall ${ }^{\# 1}$, Gaurav Sharma ${ }^{\# 1}$, Santanu Chaudhury ${ }^{* 2}$ \\ ${ }^{\#}$ Samsung India Software R\&D Centre, Logix Infotech Park, \\ D-5, Sector-59, Noida-201301, Uttar Pradesh, India, "Electrical Engineering Department, IIT Delhi \\ \{Rajen.bhatt, abhinav.d, s.gaurav1\}@samsung.com, rajen.bhatt@gmail.com, schaudhury@gmail.com
}

\begin{abstract}
We propose an efficient skin region segmentation methodology using low complexity fuzzy decision tree constructed over B, G, R colour space. Skin and nonskin training dataset has been generated by using various skin textures obtained from face images of diversity of age, gender, and race people and nonskin pixels obtained from arbitrary thousands of random sampling of nonskin textures. Compact fuzzy model with very few numbers of rules allow to raster scan consumer photographs and classify each pixel as skin or nonskin for various face and human detection applications for embedded platforms.
\end{abstract}

Keywords- Fuzzy decision trees, Skin Segmentation

\section{INTRODUCTION}

Skin-like region segmentation has been utilized as a preprocessing step for various face and human detection and tracking applications [1-6]. Colour space-based models act as efficient approaches for quickly identifying the skin-like regions before performing complicated steps like face and body detection and tracking. Various colour space-based approaches have been proposed by researchers [7-11]. However, skin region segmentation for embedded systems porting needs separate attention because of processing limitations of the devices. Further, applications in to consumer electronics products should work with very good timeaccuracy trade-off for deployment into market and success of the products. In this paper, we propose the skin-like region segmentation approach specifically for embedded systems applications with high accuracy and fast processing time as the main target. We have used fuzzy decision tree (FDT) induced over skin and non-skin training patterns. By the suitable selection of learning parameters, a compact FDT model has been generated which segments skin-like regions of consumer images in fraction of seconds.

This paper is organized as follows. In Section II, we describe the skin-like region segmentation approach proposed in this paper along with the brief description of FDT and specifically, FDT induced for the skin segmentation problem. Computational experiments and results have been discussed in Section III. Section IV concludes the paper.

\section{PROPOSED APPROACH FOR SKIn REgION SEgmeNtation}

\section{A. The Proposed Approach}

Our aim is to build an efficient human object presence algorithm and localize at least one face for categorization of consumer images into portraits and non portraits for Auto Album generation. The target processor is ARM 11 core, 500 $\mathrm{MHz}$ clock with $256 \mathrm{MB}$ DDR2 memory and two $32 \mathrm{~KB}$ cash memory. For human object detection, we have added skin segmentation as a pre-processing step followed by the other algorithms. For the induction of rule-based model for skin segmentation we have used fuzzy decision trees trained over skin and nonskin samples. We have collected skin dataset by randomly sampling $B, G, R$ values from face images of various age groups (young, middle, and old), race groups (white, black, and asian), and genders obtained from FERET database and PAL database [12,13]. Total learning sample size is 51444; out of which 14654 is the skin samples and 36790 is nonskin samples. This makes our training $\mathrm{Db}$ is of the dimension $51444 * 4$ where first three columns are B,G,R $\left(x_{1}\right.$, $x_{2}$, and $x_{3}$ features) values and fourth column is of the class labels (decision variable $y$ ). On this $\mathrm{Db}$ we have developed fuzzy decision tree using fuzzy ID3 induction algorithm. A brief explanation of fuzzy decision trees and fuzzy ID3 algorithm is given below.

\section{B. Fuzzy Decision Trees}

Fuzzy decision trees are powerful, top-down, hierarchical search methodology to extract easily interpretable classification rules $[14,15]$. Fuzzy decision trees are composed of a set of internal nodes representing variables used in the solution of a classification problem, a set of branches representing fuzzy sets of corresponding node variables, and a set of leaf nodes representing the degree of certainty with which each class has been approximated. We have used our own implementation of fuzzy ID3 algorithm $[14,15]$ for learning a fuzzy classifier on the training data. Fuzzy ID3 utilizes fuzzy classification entropy of a possibilistic distribution for decision tree generation.

Before induction of fuzzy decision tree, training patterns pertaining to three input attributes have been clustered using fuzzy $c$-means clustering algorithm [16] into five fuzzy clusters. These fuzzy clusters have been approximated as a Gaussian membership function using the dispersion factor 0.2 [17]. Plot of fuzzy membership functions after Gaussian membership estimation is shown in Fig. 1 below. 

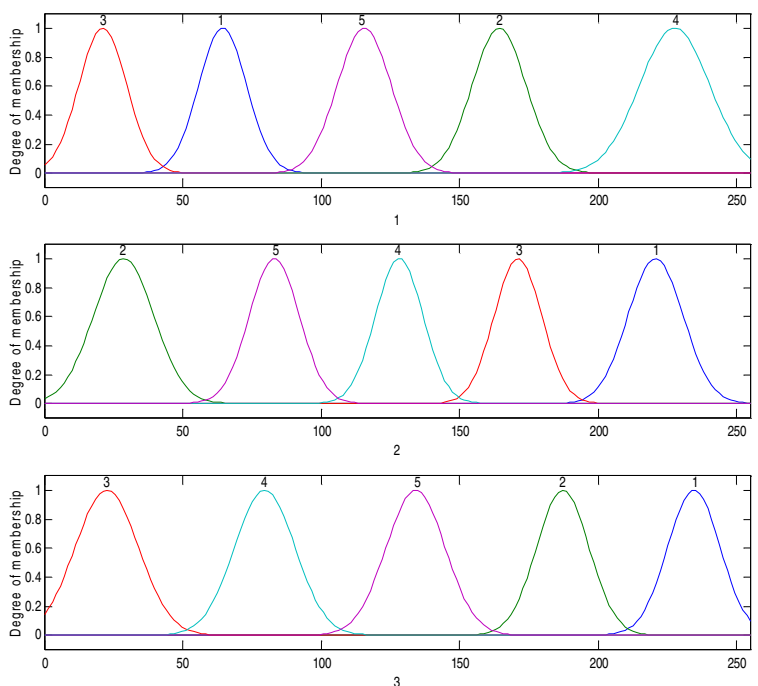

Fig. 1 Gaussian membership functions for B,G,R planes

Figure 2 shows fuzzy decision tree using fuzzy ID3 algorithm for the skin-nonskin classification problem. We have taken leaf selection threshold 0.75. In Fig. 2, root node is represented by $R=x_{3}$. There are total seven leaf nodes shown by bold dotes. Children nodes have been terminated as leaf nodes because their respective certainty thresholds (all $\beta$ ) are greater than 0.75. $\beta_{m s}$ and $\beta_{m N}$ are prediction certainties of $m^{\text {th }}$ leaf node with respect to class skin and nonskin, respectively.

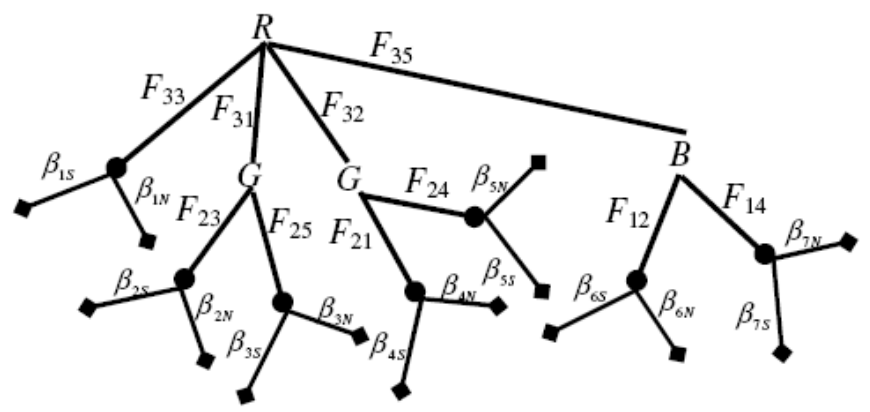

Fig. 2 Fuzzy decision tree for skin-nonskin classification problem

Certainty coefficients of all the leaf nodes are given as below :

$$
\left[\begin{array}{ll}
\beta_{1 S} & \beta_{1 N} \\
\beta_{2 S} & \beta_{2 N} \\
\beta_{3 S} & \beta_{3 N} \\
\beta_{4 S} & \beta_{4 N} \\
\beta_{5 S} & \beta_{5 N} \\
\beta_{6 S} & \beta_{6 N} \\
\beta_{7 S} & \beta_{7 N}
\end{array}\right]=\left[\begin{array}{ll}
0.00 & 1.00 \\
0.92 & 0.08 \\
0.00 & 1.00 \\
0.00 & 1.00 \\
0.94 & 0.06 \\
0.00 & 1.00 \\
0.00 & 1.00
\end{array}\right]
$$

Certainty coefficients can be calculated by standard subsethood formula [14]. For example, $\beta_{2 S}$ can be calculated by

$$
\frac{\sum_{i=1}^{n}\left(\mu_{F_{12}}\left(x_{1 i}\right) \times y_{i}\right) \times\left(\mu_{F_{12}}\left(x_{7 i}\right) \times y_{i}\right)}{\sum_{i=1}^{n} \mu_{F_{12}}\left(x_{1 i}\right) \times \mu_{F_{72}}\left(x_{7 i}\right)},
$$

where $n$ is total number of patterns, $x_{j i}$ is $i^{\text {th }}$ pattern of $j^{\text {th }}$ feature, and $\mu_{F_{i k}}\left(x_{j i}\right)$ is degree of membership of $x_{j i}$ to $k^{\text {th }}$ membership function on $j^{\text {th }}$ feature.

Using this FDT, patterns are classified by starting from the root node and then reaching to one or more than one leaf nodes by following the path of degree of membership greater than zero. One can use either min-max-max or productproduct-sum [14] reasoning mechanism over extracted rules to calculate the degree of certainty with which an arbitrary pattern can be classified to one class. In this paper, we have used the later one. The product-product-sum reasoning mechanism consists of the following three steps:

a. For the operation to aggregate membership values of fuzzy sets of node genres along the paths, the product is adopted.

b. For the operation of the total membership value of the path of fuzzy evidences and the certainty of the class attached to leaf-nodes, also the product is adopted.

c. For the operation to aggregate certainties of the same class from different paths, the sum is adopted.

To put these steps in mathematical notations, let us consider that there are total of $M$ paths of fuzzy decision tree and total number of attributes on $m^{\text {th }}$ path is $P_{m}$. With this, firing strength of $m^{\text {th }}$ path is given by

$$
\mu_{m}=\prod_{j=1}^{P} \mu_{m j}\left(x_{m j}\right) ; m=1, \ldots, M,
$$

where $x_{m j}$ is $j^{\text {th }}$ feature on $m^{\text {th }}$ path. Prediction certainty of class- 1 and class- 2 by $m^{\text {th }}$ path is given by

$$
\beta_{m 1} \times \mu_{m}, \beta_{m 2} \times \mu_{m} \text {. }
$$

Finally, aggregate the predictions certainties of class- 1 and class-2 from different paths using the following formulas.

$$
\begin{aligned}
& \hat{d}^{1}=\sum_{m=1}^{M} \beta_{m 1} \times \mu_{m} \\
& \hat{d}^{2}=\sum_{m=1}^{M} \beta_{m 2} \times \mu_{m} .
\end{aligned}
$$

The predicted class $\hat{y}$ is given by winner-takes-all logic, i.e.,

$$
\hat{y}=\max _{1 \leq q \leq Q} \hat{d}^{q} \text {. }
$$

Centre and standard deviation matrix associated with each of the path of fuzzy decision tree are given below :

$\mathbf{C}=\left[\begin{array}{ccc}0 & 0 & 22.45 \\ 0 & 170.97 & 234.49 \\ 0 & 82.95 & 234.49 \\ 0 & 220.65 & 187.17 \\ 0 & 128.06 & 187.17 \\ 164.17 & 0 & 133.97 \\ 227.50 & 0 & 133.97\end{array}\right] ; \mathbf{S}=\left[\begin{array}{ccc}0 & 0 & 11.40 \\ 0 & 8.58 & 9.46 \\ 0 & 9.02 & 9.46 \\ 0 & 9.93 & 9.46 \\ 0 & 8.58 & 9.46 \\ 9.75 & 0 & 10.63 \\ 12.66 & 0 & 10.63\end{array}\right]$.




\section{COMPUTATIONAL EXPERIMENTS}

We have performed various computational experiments on $\mathrm{PC}$ and on embedded hardware with specifications given in Section II above. Our ten fold cross validation average performance is $94.10 \%$. The average confusion matrix is given below :

$$
\text { conf }=\left[\begin{array}{ccc} 
& \text { Skin } & \text { Nonskin } \\
\text { Skin } & 98.09 & 1.90 \\
\text { Nonskin } & 7.48 & 92.51
\end{array}\right] .
$$

Above results shows that the algorithm is highly efficient in declaring actual skin as skin, where as confusion of almost $7.5 \%$ is involved for nonskin segments. We have executed the proposed algorithm for various consumer images. Some of the skin segmented and actual images are shown below for illustration. To report the timing performance all the images have been scaled to standard $640 * 480$ (i.e., VGA size) resolution.
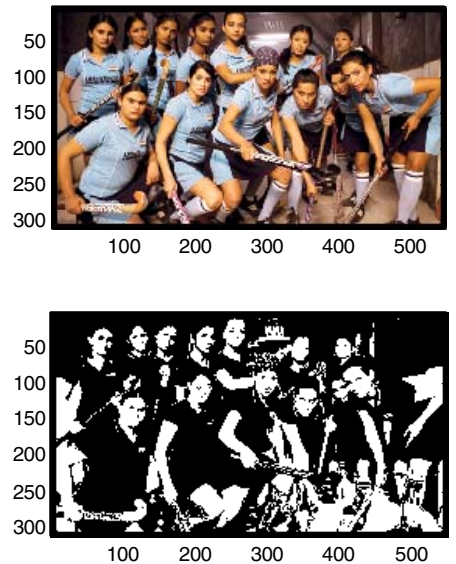

Fig. 3 Result on Chak De India group photograph. Timing $250 \mathrm{mSec}$.
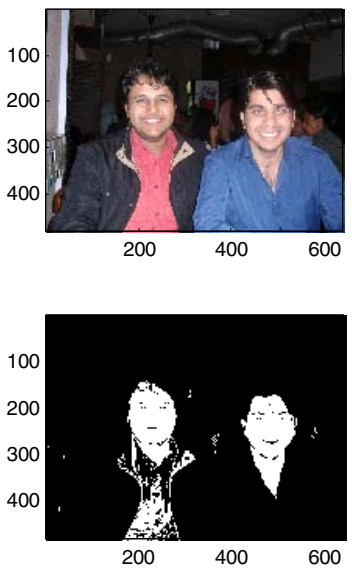

Fig. 4 Result on Asian consumer image. Timing $250 \mathrm{mSec}$.

\section{CONCLUSIONS}

In this paper, we have proposed B,G,R colour-based skin segmentation approach using fuzzy decision tree. Very compact FDT model using just seven leaf nodes (i.e., fuzzy rules) makes it very efficient for application into embedded devices. Further, each fuzzy rule makes use of at the most two attributes which makes the algorithm application fast enough for the real world applications into products.
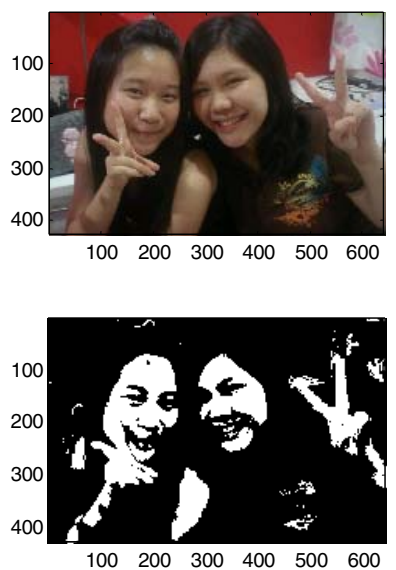

Fig. 5 Result on East Asian consumer image. Timing $216 \mathrm{mSec}$.
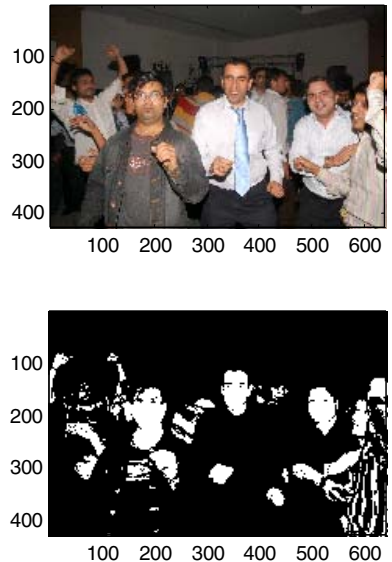

Fig. 5 Result on party photograph with varying illumination. Timing 216 $\mathrm{mSec}$.
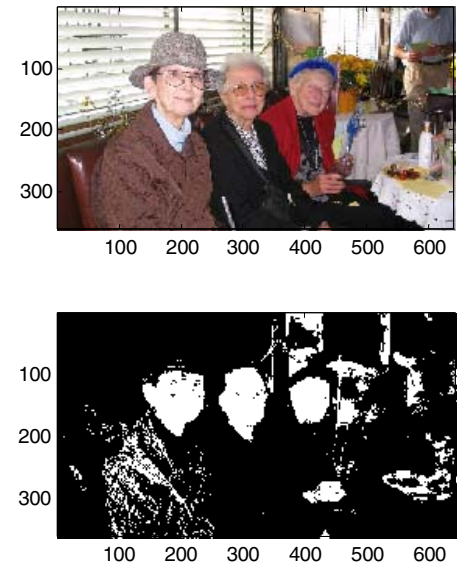

Fig. 6 Result on white race ladies. Timing $237 \mathrm{mSec}$. 

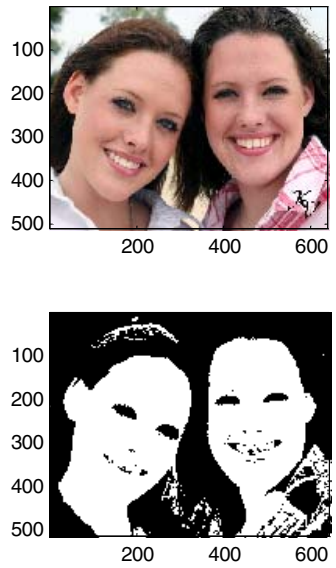

Fig. 7 Result on white race ladies portrait photograph. Timing $250 \mathrm{mSec}$.
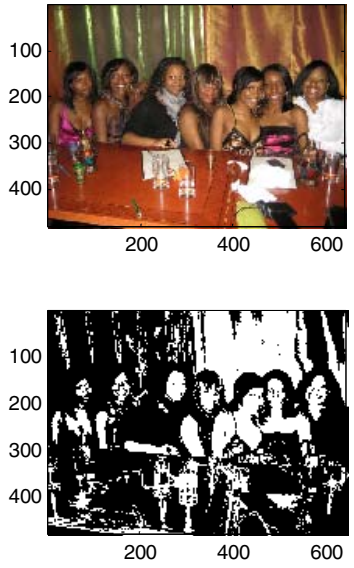

Fig. 8 Results on Black ladies with illumination. Timing $208 \mathrm{mSec}$.
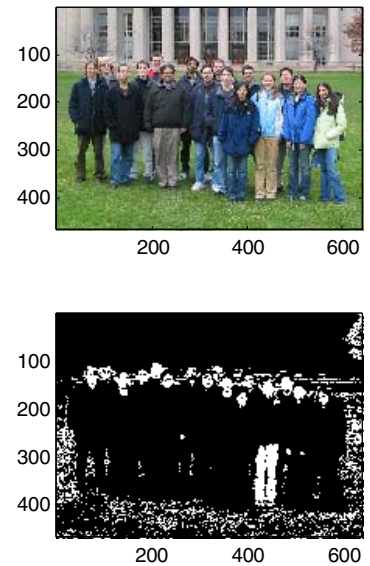

Fig. 9 Multiple faces with diversity of age, race, gender. Timing : $250 \mathrm{mSec}$

It is clearly evident from the results and timing information that at most VGA image takes $250 \mathrm{mSec}$ for the skin segmentation. As a further research, we plan to include eyelips-nose localization algorithm for complete face detection application and then detection of orientation of portrait images.

\section{ACKNOWLEDGEMENTS}

Authors are thankful to Samsung India Software R\&D Center for excellent research environment and opportunities.

\section{REFERENCES}

[1] S. Birchfield, "Elliptical head tracking using intensity gradients and color histograms" in Proc. of CVPR 1998, pp 232-237.

[2] Q. Chen, H. Wu, and M. Yachida, "Face detection by fuzzy pattern matching" in Proc. of $5^{\text {th }}$ Int. Conf. on Computer Vision, 1995, pp. 591-597.

[3] C. Wang and M. Brandstein, "Multisource face tracking with audio and visual data" in Proc. of IEEE Multimedia and Signal Processing, 1999, pp. 169-174.

[4] R.-L. Hsu, M. Abdel-Mottaleb, and A.K. Jain, "Face detection in color images", IEEE Trans. PAMI, vol. 24, no. 5, pp. 696-706, 2002.

[5] N. Oliver, A. Pentland, and F. Berard, "Lafter : Lips and Face Real Time Tracker" in Proc. of Computer Vision and Pattern Recognition, 1997, pp. 123-129.

[6] R. Schumeyer and K. Barner, "A color-based classifier for region identification in video", in Proc. of Visual Communications and Image Processing, vol. 3309, 1998, pp. 189-200.

[7] A. Albiol, L. Torres, and E.J. Delp, "Optimal color spaces for skin detection", in Proc. of Int. Conf. on Image Processing, vol. 1, 2001, pp. $122-124$

[8] J. Brand and J. Mason, "A comparative assessment of three approaches to pixel level human skin detection", in Proc. of Int. Conf. on Pattern Recognition, vol. 1, 2000, pp. 1056-1059.

[9] D. Chai and A. Bouzerdoum, "A Bayesian approach to skin color classification in YCbCr color space", in Proc. IEEE TENCON 2000, vol. 2, pp. 421-424.

[10] G. Gomez, "On selecting color components for skin detection", in Proc. of ICPR, vol. 2, 2000, pp. 961-964.

[11] J.-C. Terrillon, M.N. Shirazi, H. Fukamachi, and S. Akamatsu, "Comparative performance of different skin chrominance models and chrominance faces for the automatic detection of human faces in color images", in Proc. of Int. Conf. on Face and Gesture Recognition, 2000 , pp. 54-61.

[12] Color FERET Image Database: http://face.nist.gov/colorferet/request.html.

[13] PAL Face Database from Productive Aging Laboratory, The University of Texas at Dallas: https://pal.utdallas.edu/facedb/.

[14] X.-Z. Wang, D.S. Yeung, and E.C.C. Tsang, "A comparative study on heuristic algorithms for generating fuzzy decision trees", IEEE Transactions on SMC - B, vol. 21, no. 2, pp. 215-226, 2001.

[15] Rajen B. Bhatt, Fuzzy-rough approach to pattern classification: hybrid algorithms and optimization, $\mathrm{PhD}$ Thesis, Electrical Engineering Department, IIT Delhi, India. Call number: 621-52 BHA-F, Accession no: TH-3259, http://indest.iitd.ac.in/scripts/wwwi32.exe/[in=arphd]/.

[16] N.R. Pal and J.C. Bezdek, "On cluster validity for fuzzy $c$-means model", IEEE Transactions On Fuzzy Systems, vol. 3, no.3, pp.370379, 1995.

[17] Rajen B. Bhatt and M.Gopal, "On the structure and initial parameter identification of Gaussian RBF networks", International Journal of Neural Systems, 14 (6), pp. 1-8, 2004. 